\title{
Computational Simulations of Magnetic Particle Capture in Arterial Flows
}

\author{
J. W. Haverkort, ${ }^{1,2}$ S. Kenjerešs, ${ }^{1,2}$ and C. R. KLeiJn ${ }^{1,2}$ \\ ${ }^{1}$ Department of Multi-Scale Physics, Delft University of Technology, Prins Bernhardlaan 6, 2628 BW Delft, The Netherlands; \\ and ${ }^{2}$ J. M. Burgers Centre for Fluid Mechanics, 2628 CD Delft, The Netherlands \\ (Received 15 April 2009; accepted 26 August 2009; published online 16 September 2009)
}

\begin{abstract}
The aim of Magnetic Drug Targeting (MDT) is to concentrate drugs, attached to magnetic particles, in a specific part of the human body by applying a magnetic field. Computational simulations are performed of blood flow and magnetic particle motion in a left coronary artery and a carotid artery, using the properties of presently available magnetic carriers and strong superconducting magnets (up to $B \approx 2 \mathrm{~T}$ ). For simple tube geometries it is deduced theoretically that the particle capture efficiency scales as $\eta \sim \sqrt{M n_{\mathrm{p}}}$, with $M n_{\mathrm{p}}$ the characteristic ratio of the particle magnetization force and the drag force. This relation is found to hold quite well for the carotid artery. For the coronary artery, the presence of side branches and domain curvature causes deviations from this scaling rule, viz. $\eta \sim M n_{\mathrm{p}}^{\beta}$, with $\beta>1 / 2$. The simulations demonstrate that approximately a quarter of the inserted $4 \mu \mathrm{m}$ particles can be captured from the bloodstream of the left coronary artery, when the magnet is placed at a distance of $4.25 \mathrm{~cm}$. When the same magnet is placed at a distance of $1 \mathrm{~cm}$ from a carotid artery, almost all of the inserted $4 \mu \mathrm{m}$ particles are captured. The performed simulations, therefore, reveal significant potential for the application of MDT to the treatment of atherosclerosis.
\end{abstract}

Keywords-Magnetic drug targeting, Magnetic fields, Blood flow, Coronary artery, Carotid artery.

\section{INTRODUCTION}

The targeting of drugs to a specific region of the human body can be significantly enhanced by attaching the drugs to magnetic particles. By applying a magnetic field at the target region, the particles can be slowed down or even captured from the bloodstream. This Magnetic Drug Targeting (MDT) ${ }^{2,17-19}$ technique

Address correspondence to $\mathrm{S}$. Kenjereš, Department of Multi-Scale Physics, Delft University of Technology, Prins Bernhardlaan 6, 2628 BW Delft, The Netherlands. Electronic mail: J.W.Haverkort@cwi.nl, S.Kenjeres@tudelft.nl can significantly increase the specificity of certain medical treatments, lowering the required dose and reducing side-effects. The first clinical trials involved permanent magnets externally to the body aimed at the chemotherapeutic removal of superficial tumors. ${ }^{18}$ In order to target locations further below the skin, the use of magnetic stents ${ }^{4,10}$ and magnetic implants ${ }^{7,11,15}$ have been investigated. Two-dimensional computational simulations of magnetic particle motion in the carotid artery bifurcation have previously been performed. ${ }^{3,23}$ In this geometry, the application of MDT revealed significant potential for application to the treatment of atherosclerosis. Thrombolytic agents attached to the magnetic particles might in the future be used to dissolve plaque deposited on arterial walls. Recently also the use of superconducting magnets has been considered for MDT. ${ }^{22,27}$

In this paper, we investigate computationally if such a minimally invasive ex-vivo treatment is feasible for application to specific large human arteries. First, the blood flow and particle motion in a straight cylindrical channel and a 90 degree bended tube are analyzed. Next, unsteady simulations in the more complex geometries of a left coronary artery and a carotid artery are performed. The properties of presently available materials and magnets are used to reveal the potentials and limits of the technique. The focus on large arteries allows us to neglect diffusive phenomena, like those discussed in Grief and Richardson, ${ }^{13}$ and describe the particle motion with a discrete particle model. Enabled by the ever increasing computer power, for the first time such magnetic particle capture simulations are performed timedependently in a realistic three-dimensional domain. This constitutes a significant improvement over previous two-dimensional simulations, allowing for the incorporation of the effect of a realistic arterial geometry on the particle and fluid motion. Similar simulations are envisioned to be performed on a patient-specific basis in the near future. 


\section{MODEL EQUATIONS}

\section{Blood Flow}

The Navier-Stokes equations for an incompressible fluid $\left(\nabla \cdot \mathbf{u}_{\mathrm{f}}=0\right)$ have been solved, numerically, for the fluid velocity $\mathbf{u}_{\mathrm{f}}$ and pressure $p$

$$
\rho\left(\frac{\partial \mathbf{u}_{\mathrm{f}}}{\partial t}+\left(\mathbf{u}_{\mathrm{f}} \cdot \nabla\right) \mathbf{u}_{\mathrm{f}}\right)=-\nabla p+\nabla \cdot\left(\mu \nabla \mathbf{u}_{\mathrm{f}}\right)
$$

with $\rho$ and $\mu$ the density and dynamic viscosity of the fluid, respectively. The relative importance of inertial forces compared to viscous forces is given by the dimensionless Reynolds number $R e=\rho u_{0} l / \mu$ with $u_{0}$ and $l$ characteristic velocity and length scales of the flow under consideration. For the left main coronary artery for example $u_{0} \approx 0.1 \mathrm{~m} / \mathrm{s}$ and $l \approx 4 \mathrm{~mm}$ such that with $\mu \approx 4 \times 10^{-3} \mathrm{~m}^{2} \mathrm{~s}^{-1}$ the Reynolds number $R e=100$, which is well within the laminar flow regime.

The rheology of blood can be described by a generalized power law ${ }^{16}$

$$
\begin{aligned}
\mu & =\lambda|\dot{\gamma}|^{n-1} \\
\lambda(|\dot{\gamma}|) & =\mu_{\infty}+\Delta \mu \exp \left[-\left(1+\frac{|\dot{\gamma}|}{a}\right) \exp \frac{-b}{|\dot{\gamma}|}\right] \\
n(|\dot{\gamma}|) & =n_{\infty}-\Delta n \exp \left[-\left(1+\frac{|\dot{\gamma}|}{c}\right) \exp \frac{-d}{|\dot{\gamma}|}\right]
\end{aligned}
$$

with $|\dot{\gamma}|$ the magnitude of the strain rate. Here the values $\mu_{\infty}=0.0035$ Pas, $n_{\infty}=1, \Delta \mu=0.025$ Pas, $\Delta n=0.45$, $a=50 \mathrm{~s}^{-1}, b=3 \mathrm{~s}^{-1}, c=50 \mathrm{~s}^{-1}$, and $d=4 \mathrm{~s}^{-1}$ have been adapted from Ballyk et al..$^{5}$

\section{Particle Motion}

\section{Particle Magnetization Force}

The magnetization force $\mathbf{F}_{\mathrm{m}}$, also known as the Kelvin force or magnetophoretic force, on a magnetized particle of volume $V$ is given by

$$
\mathbf{F}_{\mathrm{m}}=\iiint_{V} \mu_{0} \mathbf{M} \cdot \nabla \mathbf{H} d V
$$

where $\mu_{0}=4 \pi \cdot 10^{-7} \mathrm{NA}^{-2}$ is the magnetic permeability of vacuum, $\mathbf{M}$ is the material's magnetization (magnetic dipole moment per unit volume), and $\mathbf{H}$ is the auxiliary magnetic field. Particles with a diameter larger than several tens of nanometers often consist of multiple magnetic domains. The magnetization of such particles can often be assumed to be approximately proportional to the applied magnetic field. Above a certain magnetic field strength the magnetization saturates to a constant value $M_{\text {sat }}$, resulting in the following model:

$$
\mathbf{M}=\left\{\begin{array}{ll}
\chi \mathbf{H} & H<M_{\text {sat }} / \chi \\
M_{\text {sat }} \hat{\mathbf{H}} & H \geq M_{\text {sat }} / \chi
\end{array} .\right.
$$

Here the proportionality constant $\chi$ is called the magnetic susceptibility. A hat is used to indicate a unit vector, i.e., $\hat{\mathbf{H}}=\mathbf{H} / H$ with $H=|\mathbf{H}|$. When the magnetic field is approximately constant over the volume $\pi D^{3} / 6$ of a spherical particle with diameter $D$ and the particle's surrounding medium has a negligible magnetic susceptibility, Eq. (3) becomes $\mathbf{F}_{\mathrm{m}}=$ $\left(\pi D^{3} / 6\right) \mu_{0} \mathbf{M} \cdot \nabla \mathbf{H}$. For a curl-free magnetic field $\mathbf{M} \cdot \nabla \mathbf{H}=M \nabla H$ such that, using Eq. (4),

$$
\mathbf{F}_{\mathrm{m}}=\left\{\begin{array}{ll}
\frac{\pi D^{3}}{6} \mu_{0} \frac{\chi}{2} \nabla H^{2} & H<M_{\mathrm{sat}} / \chi \\
\frac{\pi D^{3}}{6} \mu_{0} M_{\mathrm{sat}} \nabla H & H \geq M_{\mathrm{sat}} / \chi
\end{array} .\right.
$$

\section{Particle Trajectories}

The trajectories $\mathbf{r}(t)$ and velocities $\mathbf{u}_{\mathrm{p}}=d \mathbf{r} / d t$ of particles with mass $m$ have been calculated by integrating

$$
m \frac{d^{2} \mathbf{r}}{d t^{2}}=\mathbf{F}_{\mathrm{D}}+\mathbf{F}_{\mathrm{m}}
$$

Here $\mathbf{F}_{\mathrm{m}}$ is the magnetization force of Eq. (5). The drag force $\mathbf{F}_{\mathrm{D}}$ is modeled using the relations found in Morsi and Alexander. ${ }^{21}$ For particle Reynolds numbers $R e_{\mathrm{p}} \equiv \rho\left|\mathbf{u}_{\mathrm{p}}-\mathbf{u}_{\mathrm{f}}\right| D / \mu \ll 1$, as is generally the case for the situations studied in this work, the drag force on a spherical particle with diameter $D$ is given by the Stokes drag force

$$
\mathbf{F}_{\mathrm{D}}=3 \pi \mu D\left(\mathbf{u}_{\mathrm{f}}-\mathbf{u}_{\mathrm{p}}\right), \quad R e_{\mathrm{p}} \ll 1 .
$$

Using this expression and assuming for a moment that the fluid velocity $\mathbf{u}_{\mathrm{f}}$ and magnetization force $\mathbf{F}_{\mathrm{m}}$ are time-independent, Eq. (6) can be written in terms of the velocity difference $\Delta \mathbf{u}(t) \equiv \mathbf{u}_{\mathrm{p}}(t)-\mathbf{u}_{\mathrm{f}}$

$$
\frac{d \Delta \mathbf{u}(t)}{d t}=-\frac{\Delta \mathbf{u}(t)}{\tau}+\frac{\mathbf{F}_{\mathrm{m}}}{m} .
$$

The 'particle relaxation time' or 'particle response time' $\tau$ is given by

$$
\tau \equiv \rho_{\mathrm{p}} D^{2} / 18 \mu=\frac{D R e_{\mathrm{p}}}{18\left|\mathbf{u}_{\mathrm{p}}-\mathbf{u}_{\mathrm{f}}\right|} .
$$

Equation (8) can be solved to yield $\Delta \mathbf{u}=\Delta \mathbf{u}(t=0)$ $e^{-t / \tau}+\left(1-e^{-t / \tau}\right) \mathbf{u}_{\mathrm{m}}$, where $\mathbf{u}_{\mathrm{m}}=\mathbf{F}_{\mathrm{m}} \tau / m=\mathbf{F}_{\mathrm{m}} / 3 \pi \mu D$. The characteristic timescale $\tau=D R e_{\mathrm{p}} / 18\left|\mathbf{u}_{\mathrm{p}}-\mathbf{u}_{\mathrm{f}}\right|$ over which the particle changes its velocity becomes for $R e_{\mathrm{p}} \ll 1$ of the order of, or smaller than, the time $D / u_{\mathrm{p}}$ needed for the particle to traverse one particle diameter. When one is not interested in this short-lived transient behavior one can neglect the particle acceleration altogether. In this case $\mathbf{F}_{\mathrm{D}}+\mathbf{F}_{\mathrm{m}}=0$ or, using Eq. (7),

$$
\mathbf{u}_{\mathrm{p}}=\mathbf{u}_{\mathrm{f}}+\frac{\mathbf{F}_{\mathrm{m}}}{3 \pi \mu D}
$$


showing that the particle velocity is given by the sum of the fluid velocity $\mathbf{u}_{\mathrm{f}}$ and a 'magnetic velocity' $\mathbf{u}_{\mathrm{m}} \equiv \mathbf{F}_{\mathrm{m}} / 3 \pi \mu D=u_{0}\left(\mathbf{F}_{\mathrm{m}} / F_{\mathrm{D}}\right)$ in the direction of the magnetic force.

\section{Capture Efficiency}

A major challenge for Magnetic Drug Targeting is to create a large enough magnetic force to capture particles of reasonably small size. The material properties and magnetic field are therefore chosen such as to maximize the capture efficiency (or collection efficiency). The capture efficiency $\eta$ for a piece of artery is defined as the fraction of inserted particles that is attracted by the magnetic field towards the vessel wall and are captured or collected there. With $n_{\text {in }}$ and $n_{\text {out }}$ the number of particles entering and leaving the artery segment

$$
\eta \equiv \frac{n_{\mathrm{in}}-n_{\mathrm{out}}}{n_{\mathrm{in}}} .
$$

A short calculation serves to reveal how this important quantity $\eta$ scales with the characteristic values of parameters such as the flow velocity $\left(u_{0}\right)$, vessel radius $(R)$, and auxiliary magnetic field $(H)$. We will assume $R e_{\mathrm{p}} \ll 1$ such that $\mathbf{u}_{\mathrm{p}}=\mathbf{u}_{\mathrm{f}}+\mathbf{u}_{\mathrm{m}}$ and we simplify the analysis by assuming that $\mathbf{u}_{\mathrm{m}}$ is perpendicular to $\mathbf{u}_{\mathrm{f}}$. With $l \sim u_{\mathrm{m}} t$ the distance over which particles are displaced during a time $t \sim L / u_{0}$ in the direction of a magnetic force of spatial extent of the order $L$, one obtains a scaling for the capture efficiency $\eta \sim l / R \sim(L / R)\left(u_{\mathrm{m}} / u_{0}\right)$. Introducing the aspect ratio $\alpha \equiv L / R$ and $M n_{\mathrm{p}} \equiv u_{\mathrm{m}} / u_{0}=F_{\mathrm{m}} / F_{\mathrm{D}}$, the obtained scaling relation becomes $\eta \sim \alpha M n_{\mathrm{p}}$. The 'particle magnetization number' $M n_{\mathrm{p}}$ can be written in terms of characteristic integral quantities as

$$
M n_{\mathrm{p}}=\frac{\mu_{0} D^{2} M H}{18 \mu u_{0} L} .
$$

The above analysis assumed a uniform flow velocity $u_{0}$. To account for the typical laminar flow profile found in arteries, we linearize the flow profile close to the wall as $u_{\mathrm{f}}=u_{0}(l / R)$, with $l \ll 1$ the distance from the wall. Replacing $u_{0}$ by $u_{\mathrm{f}}=u_{0}(l / R)=u_{0} \eta$ in the scaling relation obtained from the above analysis, it follows that $\eta^{2} \sim(L / R)\left(u_{\mathrm{m}} / u_{0}\right)$ or

$$
\eta \sim \sqrt{\alpha M n_{\mathrm{p}}}, \quad \eta \ll 1 .
$$

Note that this strictly only holds for small capture efficiencies $\eta \ll 1$, for which particles are captured close to the wall where the flow velocity increases approximately linearly with the distance from the wall. The most important quantities for magnetic particle capture, and Magnetic Drug Targeting in particular, are thus found to be the ratio $M n_{\mathrm{p}}$ between the magnetization force and the drag force and the ratio $\alpha$ between the spatial extent of the magnetic field and the vessel size. Perhaps surprisingly, due to the increasing flow velocity with distance from the wall, the capture efficiency is found to depend on the square root of these quantities. This implies that a four times larger force or a four times larger spatial extent of the force is initially needed to double the capture efficiency. Equation (13) can be written in terms of characteristic integral quantities

$$
\eta \sim D \sqrt{\frac{\mu_{0}}{18 R} \frac{M H}{\mu u_{0}}}, \quad \eta \ll 1 .
$$

For undersaturated particles $M=\chi H$ such that $\eta \sim H D \sqrt{\mu_{0} \chi / 18 R \mu u_{0}}$.

\section{Materials Used}

Care has been taken to select materials that maximize the capture efficiency. Iron, which has a high magnetic susceptibility and a high saturation magnetization, has recently successfully been made into drugsusceptible carriers. ${ }^{6}$ With $67.5 \%$ iron and $32.5 \%$ carbon (by weight) these particles have a density of approximately $\rho=6450 \mathrm{~kg} / \mathrm{m}^{3}$ and a saturation magnetization of $M_{\mathrm{sat}}=10^{6} \mathrm{~A} / \mathrm{m}$. The properties of these particles were used in the simulations. Because saturation occurs even for fields as low as $0.05 \mathrm{~T}$, the particles were assumed to be saturated in the entire domain.

For the magnetic field we used the properties of a cylindrical superconducting magnet with a diameter of $4.5 \mathrm{~cm}$, a thickness of $1.5 \mathrm{~cm}$ and a maximum field strength of $B \approx 2 \mathrm{~T}$, as reported in Takeda et al. ${ }^{27}$ Owing to its physical origin, the associated magnetic field resembles that of circular line current. For a loop of radius $a$, carrying a current $I$, with $H_{0} \equiv I / 2 a$ the magnetic field is given by ${ }^{25}$

$$
\begin{aligned}
\mathbf{H}\left(z^{\prime}, s^{\prime}\right)= & \frac{H_{0}}{\pi \sqrt{\left(1+s^{\prime}\right)^{2}+z^{\prime 2}}}(K(k)(\hat{\mathbf{z}}-\hat{\mathbf{s}}) \\
& \left.+E(k) \frac{1-s^{\prime 2}-z^{\prime 2}}{\left(1+s^{\prime}\right)^{2}+z^{\prime 2}-4 s^{\prime}}(\hat{\mathbf{z}}+\hat{\mathbf{s}})\right) .
\end{aligned}
$$

Here $K(k)$ and $E(k)$ are elliptic integrals of the first and second kind, ${ }^{1}$ respectively, with the argument $k$ given by

$$
k=\sqrt{\frac{4 s^{\prime}}{\left(1+s^{\prime}\right)^{2}+z^{\prime 2}}} .
$$

The cylindrical coordinates $z$ and $s$ represent the distance parallel and perpendicular to the symmetry axis, respectively (see Fig. 1). A prime denotes 


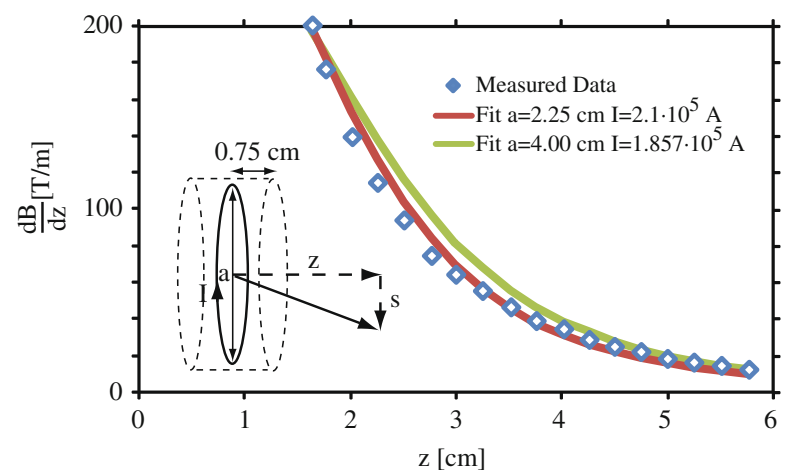

FIGURE 1. A comparison between the measured magnetic field gradient $d B / d z$ of a cylindrical superconducting magnet ${ }^{27}$ and two fits using the field $B=\mu_{0} H=\mu_{0} / / 2 a\left(1+(z / a)^{2}\right)^{3 / 2}$ of a circular loop of radius a carrying a current $l$.

nondimensionalization with the loop radius $a$, i.e., $s^{\prime} \equiv s / a$ and $z^{\prime} \equiv z / a$. A hat is again used to denote a unit vector. The field on the symmetry axis is given by $H=H_{0} / 2 a\left(1+z^{\prime 2}\right)^{3 / 2}$, directed towards the center of the loop. At a distance $z$ of several centimeters, a good fit to the reported data is obtained using a current $I=2.1 \cdot 10^{5} \mathrm{~A}$ through a loop of diameter $2 a=4.5 \mathrm{~cm}$, located halfway the cylindrical magnet, i.e., $7.5 \mathrm{~mm}$ below its upper surface as shown schematically in Fig. 1. For smaller distances, however, a current $I=1.857 \cdot 10^{5}$ A through a loop of $2 a=4 \mathrm{~cm}$ in diameter provides a better fit. The first of these fits was used for the coronary artery simulations while the latter was used for the carotid artery. Both of these fits are compared with the reported field gradient of Takeda et al. ${ }^{27}$ in Fig. 1.

\section{NUMERICAL SOLUTION METHODS}

The fluid flow and particle equations were solved using Fluent 6.3 by ANSYS, Inc., which is a finite-volume based solver for fluid flows in general geometries. User-defined functions were written to implement the viscosity model of Eq. (2) and the particle magnetization force of Eq. (5) for the field of Eq. (15). Equation (1) was solved implicitly with a quadratic upwind discretization (QUICK) of the nonlinear term. Equation (6) was numerically integrated using a sixth order Runge-Kutta scheme whenever the left hand side was significant, and an implicit Euler scheme otherwise. With a typical particle relaxation time (see Eq. 9) of $\tau=\rho_{\mathrm{p}} D^{2} / 18 \mu \approx 10^{-7} \mathrm{~s}$ for a $1 \mu \mathrm{m}$ particle the implicit Euler scheme was used most of the time. The time step for the fluid flow was chosen to be $1 / 100$ th of the flow period. The particle time step was dynamically chosen to yield a given tolerance. Alternative schemes, various time step sizes and accuracy options were tested with no appreciable differences, providing confidence in the accuracy of the integration. To validate our implementation, the Fluent simulation results were compared to results from identical simulations using an in-house fluid and particle code. ${ }^{8}$ In addition a comparison with analytical expressions has been performed to further validate the implementation. ${ }^{14}$

Particles with diameters of $250,500 \mathrm{~nm}, 1,2$, and $4 \mu \mathrm{m}$ were inserted into the flow, homogeneously distributed over the inlet. Simulations with a periodic time-dependent velocity inlet were performed in which particles were inserted at consecutive time intervals of $1 / 10$ th of the flow period, in order to be able to average over a flow cycle. Per particle size per injection approximately 260 particles were inserted. The particles were made to elastically collide with the arterial wall. In the case of the carotid artery an inelastic boundary condition, in which the particle velocity vanishes after impact, was also tested. The exact interaction of magnetic particles with the endothelial lining is much more complicated than such a simple boundary condition does justice to, see, e.g., Decuzzi et al. ${ }^{9}$ In the present simulations however, the specific boundary conditions do not strongly influence the results.

\section{RESULTS}

First, the fluid and particle motion in a straight cylindrical channel and a ninety degree bended tube is computed. These simulations serve to illustrate several features also arising in more complex arterial configurations. Thereafter, results for the left coronary artery and the carotid artery are discussed.

\section{Ninety Degree Bended Tube}

The studied geometry is shown in Fig. 2. A tetrahedral multi-block mesh was used, containing approximately 112,000 nodes. The 90 degree bend, with circular cross-section of radius $R=3.5 \mathrm{~mm}$ and

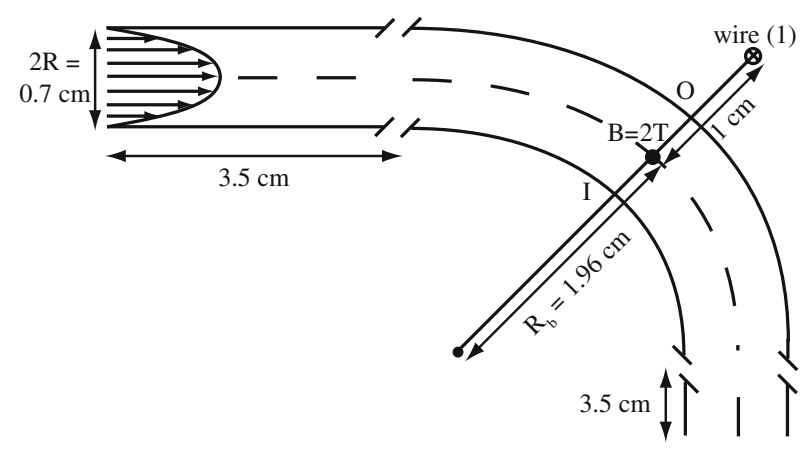

FIGURE 2. A schematic overview of the geometrical parameters used for the 90 degree bended tube. 

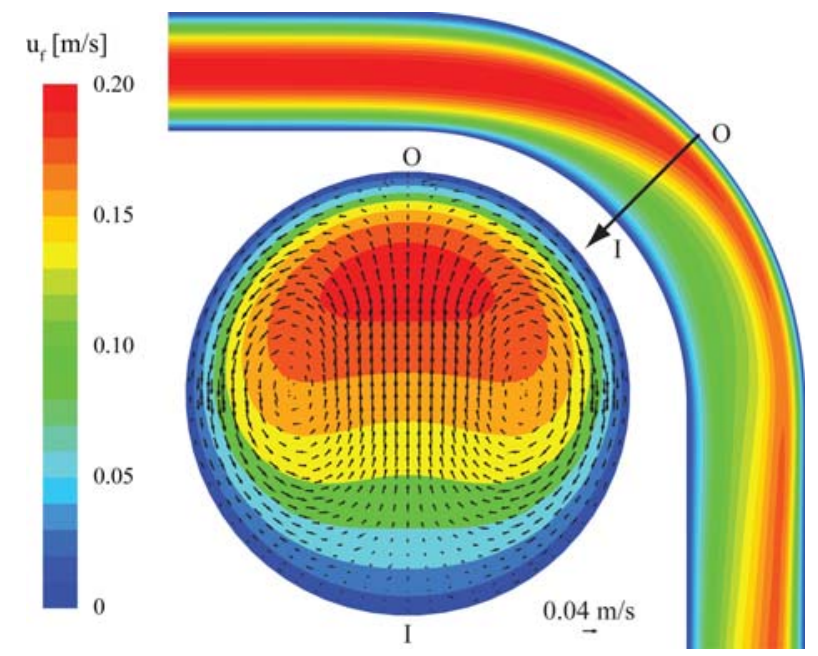

FIGURE 3. The velocity magnitude (contours) and secondary velocity (vectors) in the simulated bended tube. The maximum secondary flow velocity is $40 \%$ of the average flow velocity.

radius of curvature $R_{\mathrm{b}}=1.96 \mathrm{~cm}$, is preceded and followed by $3.5 \mathrm{~cm}$ of straight tube. With an average inlet velocity of $u_{0}=0.1 \mathrm{~ms}^{-1}$ and a constant dynamic viscosity $\mu=3.5 \cdot 10^{-3}$ Pas the Reynolds number $R e=200$ and the Dean number $D e \equiv R e \sqrt{R / R_{\mathrm{b}}}=$ 84.5 , allowing for a stable solution without flow separation. These conditions represent a physiologically quite high curvature case similar to those of the coronary artery bend.

The fluid velocity, resulting from the simulations, is shown in Fig. 3. The presence of two characteristic counter-rotating Dean-vortices can clearly be seen. The centrifugal force in the fast flowing center of the channel forces the fluid outwards. By continuity, a counteracting inwards pressure force arises, forcing the fluid to flow back through the more viscous region near the sidewalls. Consequently the maximum flow velocity is shifted from the center of the flow towards the outer curvature of the bend, as can be seen from Fig. 3.

For instructive purposes we first consider the magnetic particle motion in the magnetic field of an infinitely long straight wire carrying a current $I$. The magnitude of the field at a distance $s$ from the wire is given by $H=I / 2 \pi s$. The associated force on an undersaturated spherical particle is, using Eq. (5), given by $\mathbf{F}_{\mathrm{m}}=\mu_{0} \chi I^{2} D^{3} \nabla s / 24 \pi s^{3}$ in the direction $\nabla s$ of the wire. Various wire positions have been investigated which are, along with the magnetization force they produce, schematically depicted in Fig. 4. For all configurations the distance of closest approach from the wire to the centerline of the tube was chosen to be $1 \mathrm{~cm}$. For configuration 1 this is shown in Fig. 2. A current $I=10^{5}$ A was chosen, yielding a magnetic field

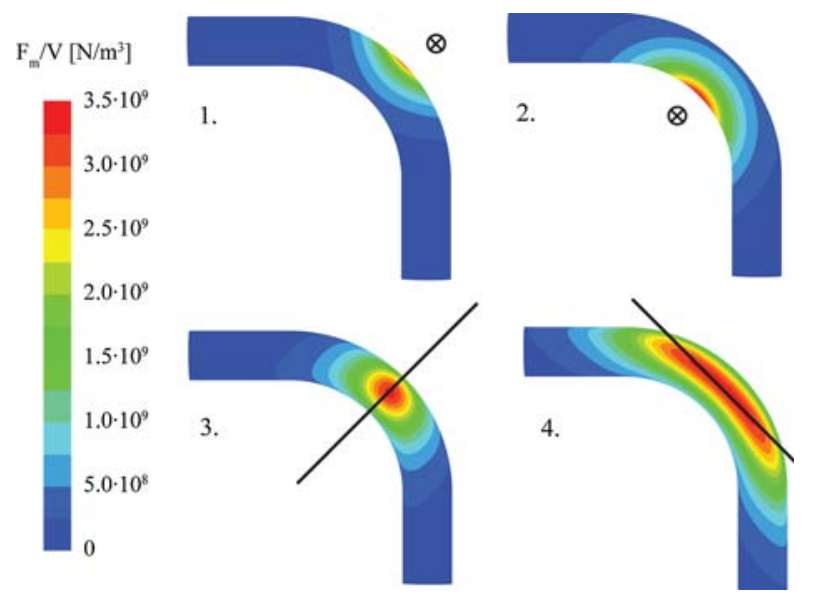

FIGURE 4. A schematic overview of the various considered wire configurations and the corresponding particle magnetization force per unit volume $F_{\mathrm{m}} / V$ for undersaturated particles with a magnetic susceptibility $\chi=3$.

of $B=\mu_{0} H=2 \mathrm{~T}$ on the tube centerline halfway the bend.

For each of the five studied particle sizes (see section "Numerical Solution Methods") $n_{\text {in }} \approx 350$ particles were inserted into the fluid flow, homogeneously distributed over the inlet. By counting the number of particles $n_{\text {out }}$ escaping through the outlet, the capture efficiency $\eta$ was obtained from Eq. (11). For comparison, the same simulation was performed using an in-house fluid and particle code ${ }^{8}$ using $n_{\text {in }}=10,000$ particles. The results of these simulations are shown in Fig. 5a. Because at most a few percent difference between the results from the in-house code and the results from Fluent was found, for clarity the latter results are left out in this figure. Given the small difference between these results, the simulations in the rest of this paper have been performed using Fluent. The different curves in Fig. 5a correspond to the different wire configurations of Fig. 4. Although the magnitude of the magnetization force halfway the bend is the same in all cases, there is a large variation in the capture efficiency. This can be attributed to geometrical differences. Notice, for example, from Fig. 4 that configuration 4 has the largest area over which the magnetization force is appreciable, explaining why for this configuration the most particles are captured.

For comparison, the exact same simulation was performed for a straight cylindrical domain, resulting in a parabolic Poiseuille flow. The current carrying wire was oriented at a right angle to the flow. For a straight channel the capture efficiency was found to vary approximation linearly with the particle diameter, as can be seen from the dashed curve in Fig. 5a, obtained with Fluent. Because the particle Reynolds 

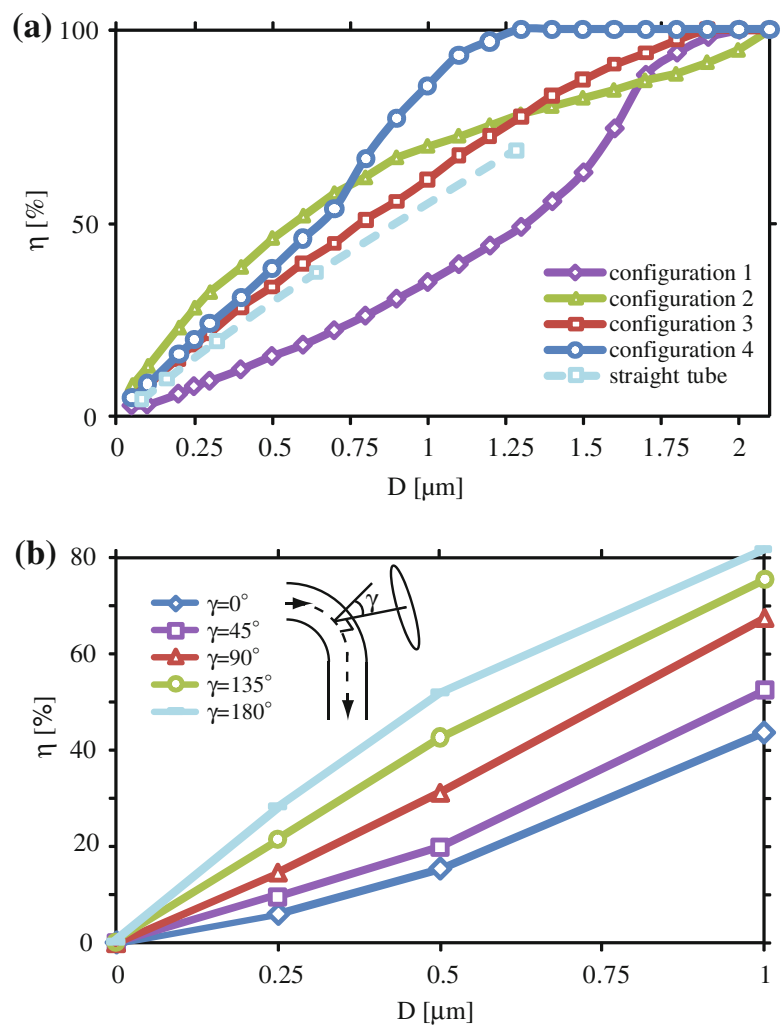

FIGURE 5. The particle capture efficiency $\eta$ as a function of the particle diameter $D$ for (a) the different current carrying wire configurations of Fig. 4 as well as for a straight tube and (b) for various angles $\gamma$ of a circular current loop. Note that in the figure both the size of the current loop and its distance to the channel centerline are not drawn to scale. Note also that these results, except for the four wire configurations in (a) which have been obtained using an in-house code, have been obtained with Fluent.

number $R e_{\mathrm{p}} \ll 1$, the only relevant non-geometrical parameter involved is $M n_{\mathrm{p}}$ which is, from Eq. (12), proportional to $D^{2}$. The obtained proportionality $\eta \sim D$, therefore, implies $\eta \sim \sqrt{M n_{\mathrm{p}}}$. The simulations of a straight cylindrical channel therefore confirm Eq. (13) and show that it continues to hold for moderate capture efficiencies. This result for a straight geometry has important consequences for magnetic drug targeting. It shows that the capture efficiency is less sensitive to the ratio between the magnetization force and the drag force than one might expect. In order to double the capture efficiency, a doubling of the magnetization force does not suffice. The obtained scaling shows that a four times higher magnetization force is required to double the capture efficiency. On the other hand when the flow velocity becomes four times as high, the capture efficiency is only approximately halved. In section "Capture Efficiency" the origin of this nonlinear behavior was shown to be due to the approximately linear increase of the flow velocity at small distance from the wall. To double the capture efficiency, particles have to be captured from twice as far where the particles move with a twice as high flow velocity, thereby requiring a four times higher force.

The spatial force distribution inside the straight channel is very similar to that of configuration 3 of the bended tube. Correspondingly, the capture efficiency of this configuration is very similar to that obtained in a straight channel. Apparently the altered flow distribution in the bended tube, compared to the parabolic flow profile in a straight tube, does not lead to large differences in the capture efficiency. For configuration 4, the capture efficiency also increases approximately linearly with the particle diameter. For configurations 1 and 2, however, this linear relationship ceases to hold. The observation that for configuration 2, for which the magnetic source is placed on the inside of the bend, it becomes increasingly difficult to capture particles of larger size seems to suggests an involvement of the centrifugal force. The ratio between the centrifugal force and the drag force is, however, only of the order $\left(m u_{0}^{2} / R_{\mathrm{b}}\right) / 3 \pi \mu u_{0} D=\rho_{\mathrm{p}} D^{2} u_{0} / 18 \mu R_{\mathrm{b}}=O\left(10^{-6}\right)$ for particles with a diameter of $D=2 \mu \mathrm{m}$. Rather, the observed trend can be explained from the flow distribution. As can be seen from Fig. 3, the fluid near the outside of the bend is flowing much faster than the fluid near the inside of the bend. To increase the capture efficiency, particles have to be attracted from further away. For a wire at the inside of the bend this means capturing particles from a region with a higher flow velocity. For particles at the outside of the bend this means capturing particles from a region of lower flow velocity. This explains the convex and concave shape of the capture efficiency curves in Fig. 5a for configurations 1 and 2, respectively. When all particles are captured, this consequence of the velocity distribution approximately levels out. The two capture efficiency curves therefore approach each other again when $\eta$ approaches $100 \%$. For the wire at the inside of the bend, a slightly higher particle diameter is needed to capture all particles than for a wire at the outside of the bend, even though the force distribution is somewhat more favorable for particle capture for the former configuration. This can be explained by the fact that the secondary flow velocity, displayed in Fig. 3, is mostly directed outwards. For the wire at the inside of the bend, the secondary flow velocity primarily adds negatively to the particle's magnetic velocity, thereby decreasing the capture efficiency.

The same simulation has been performed using the magnetic field of a circular current loop, Eq. (15). A loop with radius $a=1 \mathrm{~cm}$, carrying a current $I=3 \times$ $10^{5} \mathrm{~A}$, was placed at a distance of $1 \mathrm{~cm}$ from the centerline halfway the bend. Fully saturated particles 
were inserted with a saturation magnetization of $M_{\text {sat }}=10^{6} \mathrm{Am}^{-1}$. Systematically the angle $\gamma$ between the axis of the current loop and the plane of the bend, as shown in Fig. 5b, was varied from the outside of the bend $\left(\gamma=0^{\circ}\right)$ to the inside of the bend $\left(\gamma=180^{\circ}\right)$. The results are shown in Fig. 5b. Although admittedly few data points have been used in this graph, the trend is clear. It can be seen that for $\gamma=45^{\circ}$ the capture efficiency varies approximately linearly with the particle diameter, or $\eta \sim \sqrt{M n_{\mathrm{p}}}$. Again the effect of the flow distribution is reflected in the shapes of the curves for the other orientations.

\section{Left Coronary Artery}

For the left coronary artery a geometry was used that was obtained from the average data of 83 angiographies from healthy patients. Details can be found in Giannoglou et al. ${ }^{12}$ A steady solution independent of a further increase in the number of nodes was reported, depending on the specific criteria used, for a mesh with 60,000 or 80,000 nodes. Mesh independence is an important criterion for obtaining accurate flow solutions, ensuring the simulations resolve the important flow features without much numerical diffusion altering the solution. We used a slightly under-resolved mesh containing approximately 44,000 nodes and 200,000 cells. This can be defended on the basis that we are not interested in the detailed flow pattern but in the effect of a reasonable average flow on the particle motion. As discussed in section "Discussion and Conclusions" various other approximations made in the simulations are arguably more important than numerical errors. The computational domain (see Fig. 7) includes the largest side branch of the coronary artery, the circumflex artery, and several other large side branches. The left main coronary artery, with an initial diameter of $2 R=4.5 \mathrm{~mm}$ evolves into the left anterior descending artery with a final diameter of $0.9 \mathrm{~mm}$. A flattened radial velocity profile $u_{\mathrm{f}}(r, t)=$ $(4 / 3) u_{\text {in }}(t)\left(1-(r / R)^{6}\right)$ was used as an inlet boundary condition, where $u_{\text {in }}(t)$ is given by the characteristic pulsating flow profile displayed in Fig. 6. The average Reynolds number based on inlet parameters is approximately $R e \approx 100$. The flow rate was forced to distribute itself over the several outlets according to Murray's law, ${ }^{24}$ i.e., proportional to the third power of the outlet radii. The outlet radii and the corresponding fraction of the total flow rate per outlet, following from Murray's law, are reported in Soulis et al. ${ }^{26}$

A superconducting cylindrical magnet, modeled by a circular current loop (see section "Materials Used"), was in the simulations positioned approximately at the patient's chest. The magnet's symmetry axis was directed towards the target location, located opposite

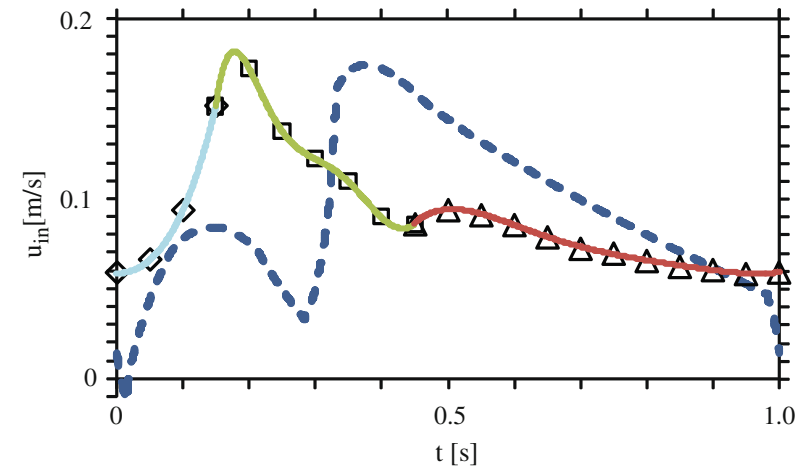

FIGURE 6. The used inlet velocity $u_{\text {in }}$ for the left coronary artery (dashed line) and for the carotid artery (solid line), which is a fit to the data points of Marshall et al. ${ }^{20}$

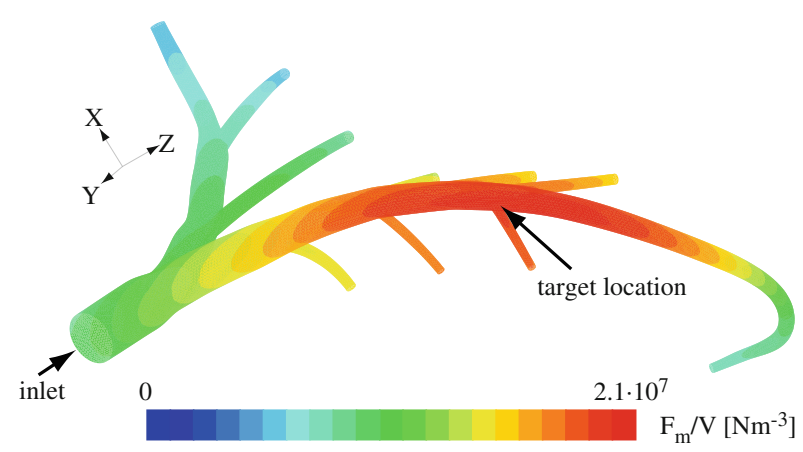

FIGURE 7. The magnitude of the magnetization force per unit volume $F_{\mathrm{m}} / V$ at the surface of the left coronary artery for the magnet oriented in the direction $(1,-2,-1)$ (configuration 2).

various side branches, as indicated by the arrow in Fig. 7. This location is a well-known region of low endothelial shear stress, which correlates with the formation of atheromatous plaques. ${ }^{26} \mathrm{~A}$ possible future application of the simulated technique could be the magnetic targeting of thrombolytic drugs, able to dissolve a plaque, in order to prevent a threatening plaque rupture. Three different magnet orientations were investigated, while the distance from the magnet's center to the target location was kept fixed at $z=5 \mathrm{~cm}$. For configurations 1,2 , and 3 the axis of the magnet was, in the coordinates of Figs. 7 and 8, directed in the $(1,-3,-1),(1,-2,-1)$, and $(1,-2,-2)$ direction, respectively. The magnetic field associated with configuration 2 is shown in Fig. 7.

Figure 8 shows stream traces for two time instances during the cardiac flow cycle, which is displayed in Fig. 6. Note that $t=0 \mathrm{~s}$ corresponds to a period of deceleration in which an adverse pressure gradient exists, causing some flow reversal.

Particles of various sizes were inserted through the inlet, at several instances during one flow cycle (as discussed in section "Numerical Solution Methods"). 
The resulting particle distributions at several time instances, after the first injection at $t=0 \mathrm{~s}$, are displayed in Fig. 9. Contrary to the simulations

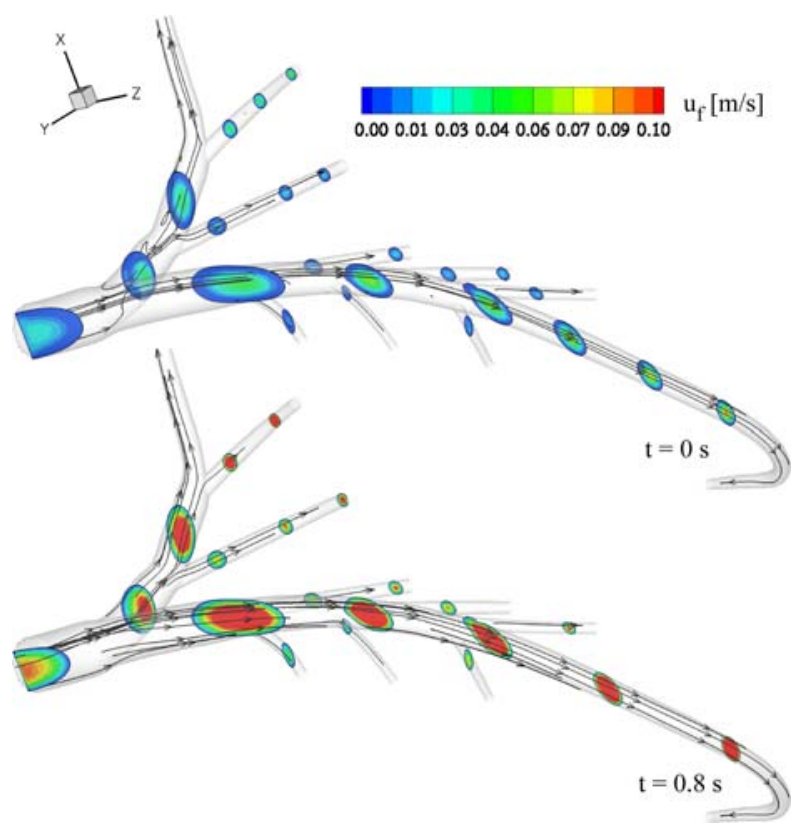

FIGURE 8. Stream traces and contours of the velocity magnitude $u_{f}$ in the left coronary artery at two different time instances. Note that several computational flow cycles preceded $t=0 \mathrm{~s}$, in order to arrive at a solution not changing from one flow cycle to the next. performed in the absence of a magnetic field, several flow seconds after the first injection a significant fraction of the particles was still present in the domain when a magnetic field was applied. Under the influence of the magnetization force these particles will remain in the domain as long the magnetic field is applied.

The fraction of injected particles that are captured is displayed in Fig. 10a for the different orientations of the magnetic field. A significant fraction of the $4 \mu \mathrm{m}$ particles is captured, constituting a positive result for the ex-vivo application of MDT to large arteries. Also note from Fig. 10a the super linear increase of the capture efficiency $\eta$ with particle diameter $D$, contrasting with the result $\eta \sim \sqrt{M n_{\mathrm{p}}} \sim D$ previously obtained for a Poiseuille flow. Upon doubling the particle size from 2 to $4 \mu \mathrm{m}$, the capture efficiency approximately quadruples. This is similar to what was found in the previous section when a magnetic source was placed on the outside of a 90 degree bended tube. Because in these simulations the relevant parameters were comparable to those of the left coronary artery, the same underlying mechanism might indeed play a role. The effect in the case of the left coronary artery is however much more pronounced and part of its origin finds its reason in the following. Figure 10b shows the fraction $\delta$ of the particles that escape through the circumflex side branch. Without an applied magnetic field, this fraction was approximately $\delta=50 \%$,

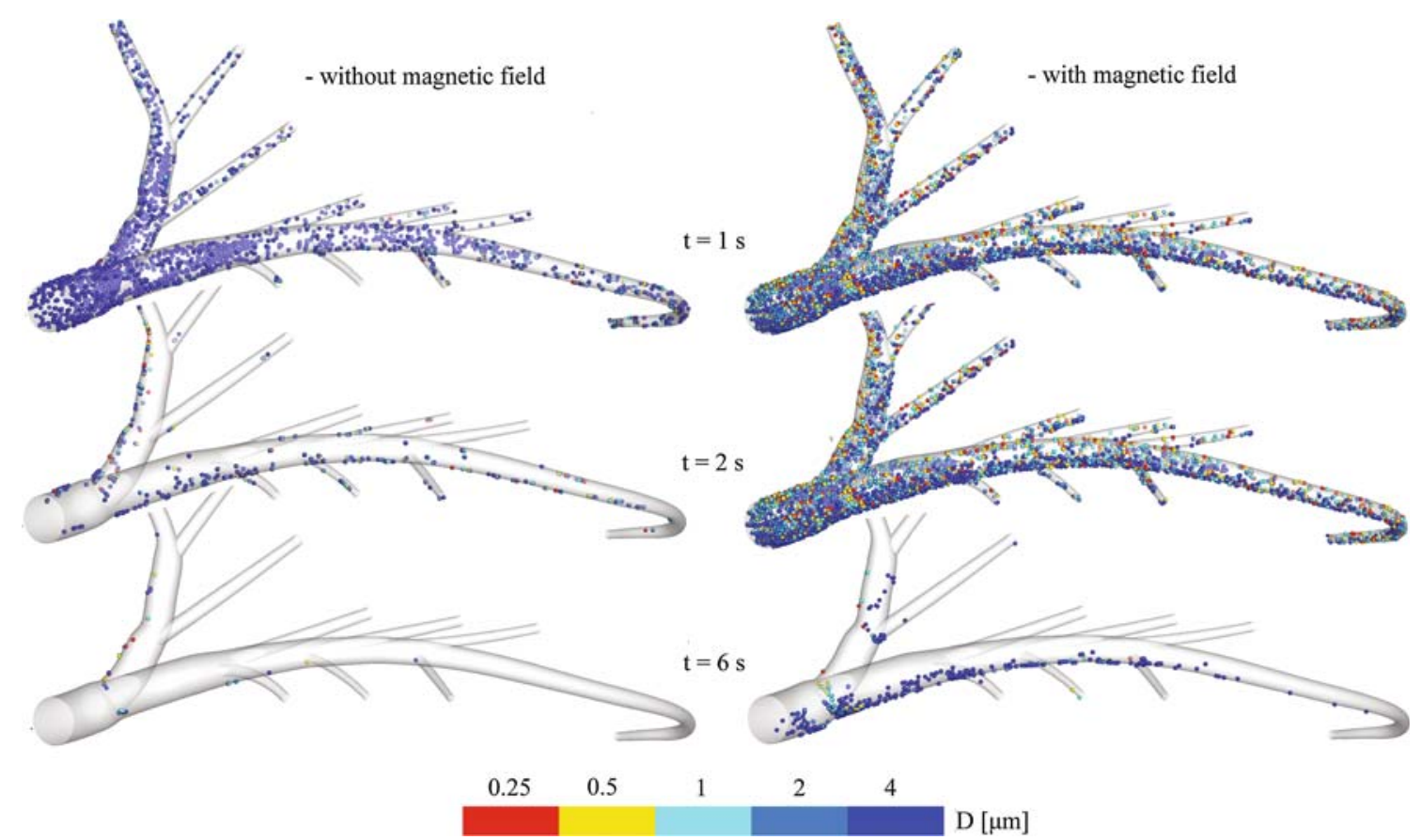

FIGURE 9. Particle distribution in the left coronary artery, at several time instances after the first injection of particles. Note that the particle sizes are not drawn to scale. In the absence of a magnetic field the trajectories of particles with a different diameter initially overlap due to negligible inertia. When the magnetic field of Fig. 7 is applied, the larger particles are attracted towards the side where they can exchange attached drugs with the arterial wall. 
irrespective of particle size. When a magnetic field is applied this fraction decreases significantly for the larger particles. Particles that would otherwise have left the domain through the circumflex sidebranch are now either attracted into the left main coronary artery where they are possibly captured, or captured in the circumflex sidebranch as can be seen in Fig. 9. Both of these effects add to the capture efficiency. Because these effects only occur for the larger particles, for which the magnetization force is large enough, it helps explaining the super linear increase of the capture efficiency with particle diameter $D$. Note that for smaller particles, the fraction of particles $\delta$ leaving through the circumflex artery actually increases. This is mostly due to particles near the wall opposite the target region, which in the absence of a magnetic field would have slowly moved into the main coronary artery. Through the action of the magnetization force, however, these particles enter a region of faster flowing fluid where they have a chance of ending up in the circumflex artery.

\section{Carotid Artery}

The carotid artery geometry was obtained from an MRI scan of a single patient with a mild stenosis at the location of the carotid sinus. ${ }^{28}$ An unstructured tetrahedral mesh was generated with 53,000 nodes and 263,000 cells, which is shown in Fig. 11a.

Average data of 14 healthy carotid arteries ${ }^{20}$ was used to make a piecewise polynomial of the flow rate $q(t)$ in $\mathrm{mL} / \mathrm{s}$ :
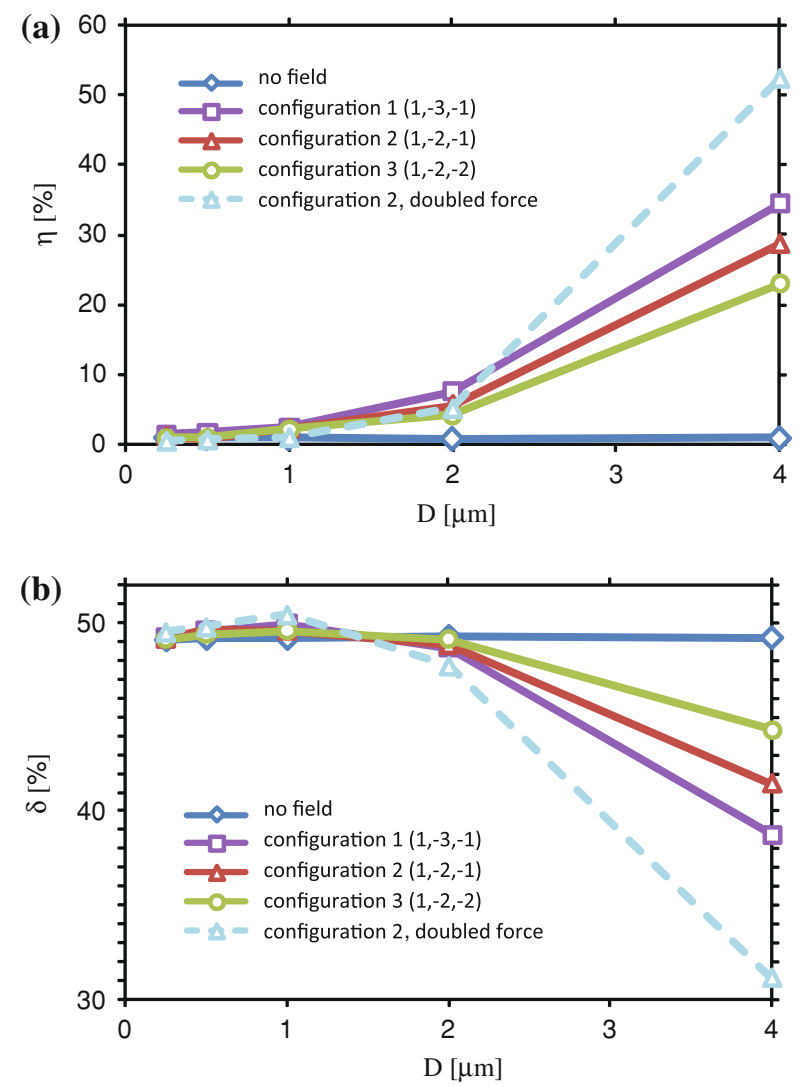

FIGURE 10. Results for the left coronary artery of (a) the capture efficiency $\eta$ as a function of particle diameter $D$ for various magnetic field orientations and (b) the fraction $\delta$ of injected particles that leave the domain through the circumflex artery or one of its side branches. The magnetic field of configuration 2 is shown in Fig. 7.

$$
q(t)= \begin{cases}946.67 t^{3}+130 t^{2}+0.9333 t+3.94 & 0 \mathrm{~s} \leq t<0.15 \mathrm{~s} \\ -424889 t^{6}+876000 t^{5}-732289 t^{4}+317210 t^{3}-74927 t^{2}+9100.3 t-430.54 & 0.15 \mathrm{~s} \leq t \leq 0.45 \mathrm{~s} \\ -601.31 t^{6}+3582.2 t^{5}-8384.8 t^{4}+10028 t^{3}-6510.4 t^{2}+2177.1 t-286.61 & 0.45 \mathrm{~s} \leq t<1 \mathrm{~s}\end{cases}
$$

This fit was used to vary in time the flow rate of a parabolic inlet velocity profile. The average Reynolds number based on inlet parameters is approximately $R e \approx 200$. An average flow distribution of approximately $70 \%$ to the internal and $30 \%$ to the external carotid arteries has been reported, ${ }^{20}$ which was used as an outlet boundary condition. The target location, indicated by the black arrow in Fig. 12, was chosen at the carotid bifurcation which is usually located only a small distance below the skin. The distance between the surface of the magnet and the target location was chosen between 1 and $2 \mathrm{~cm}(z=1.75$ and $2.75 \mathrm{~cm}$, see section "Materials Used"). The symmetry axis of the cylindrical magnet was directed normal to the plane of the bifurcation, resulting in the distributions of the magnetization force per unit volume $\mathbf{F}_{\mathrm{m}} / V$ shown in Fig. 12. In this figure, apart from the magnitude $F_{\mathrm{m}} / V$, also the radial s-component $F_{\mathrm{ms}} / V$, is shown. Here the cylindrical coordinate $s$ is the distance to the axis of symmetry of the cylindrical magnet.

Figure $11 \mathrm{~b}$ shows stream traces and velocity magnitude contours at one instant during the flow cycle. Particles of various sizes were inserted, spread over one flow cycle (see section "Numerical Solution Methods"). Figure 13 shows how a large fraction of these particles accumulate on the arterial wall. The magnetic field configuration corresponding to this simulation results is shown in Fig. 12. The capture efficiency as a 
(a)

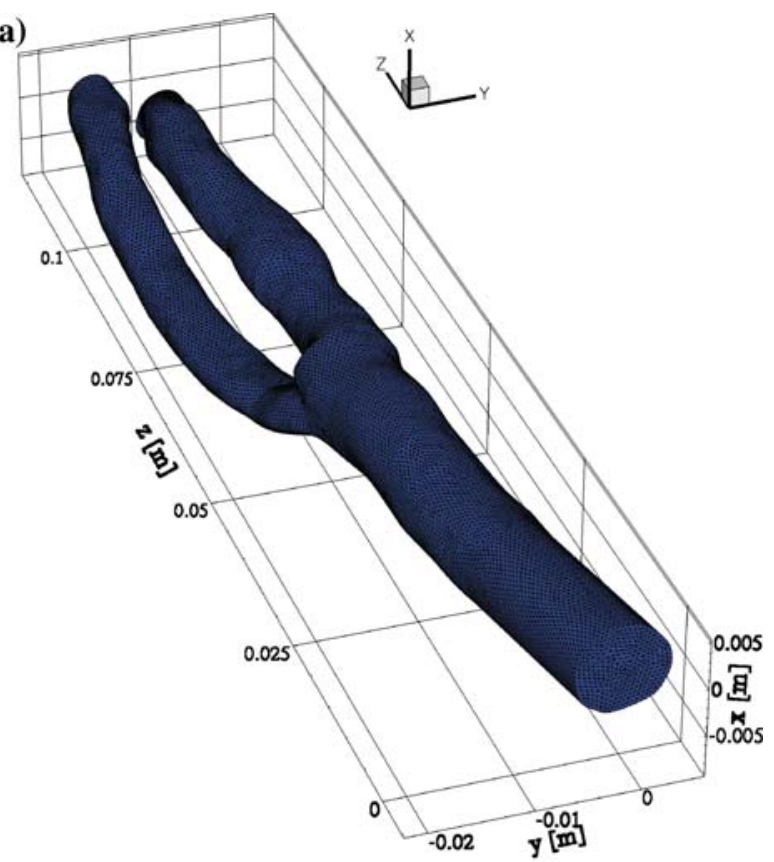

(b)

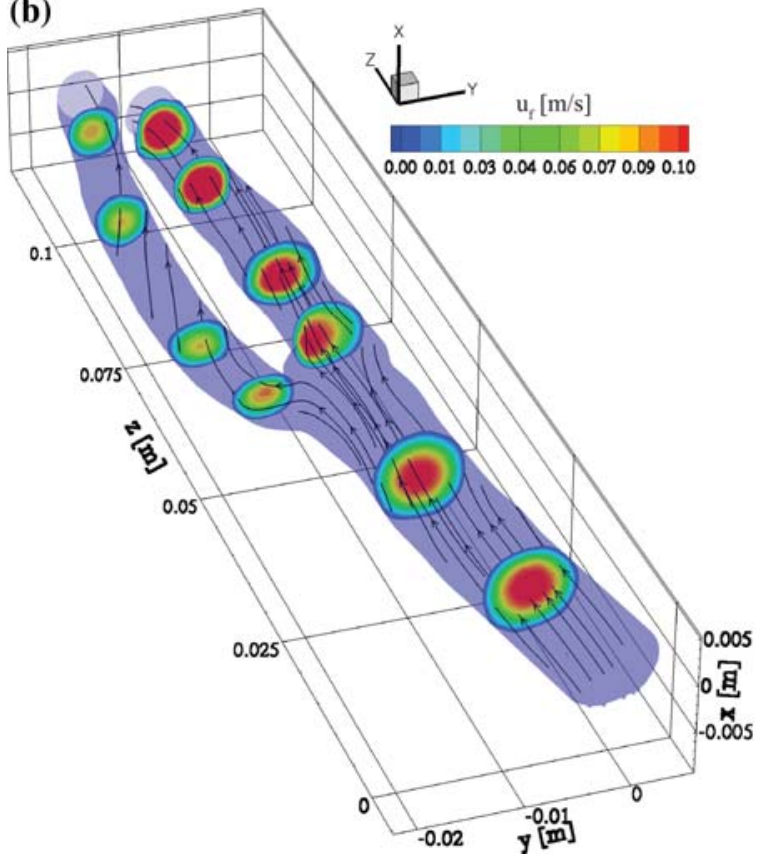

FIGURE 11. (a) The fully unstructured tetrahedral mesh of a carotid artery. At the beginning of the internal carotid artery (right branch) a dent can be seen at the location of the carotid sinus, signaling the presence of plaque. (b) Flow stream traces together with velocity magnitude contours in the carotid artery geometry at $t=0.8 \mathrm{~s}$. A skewed velocity profile can clearly be seen in the internal carotid artery just after the carotid bifurcation.

function of particle size is displayed in Fig. 14, showing that almost all $4 \mu \mathrm{m}$ particles were captured when the magnet was placed at $1 \mathrm{~cm}$ from the carotid bifurcation. This much closer proximity of the magnet (a)
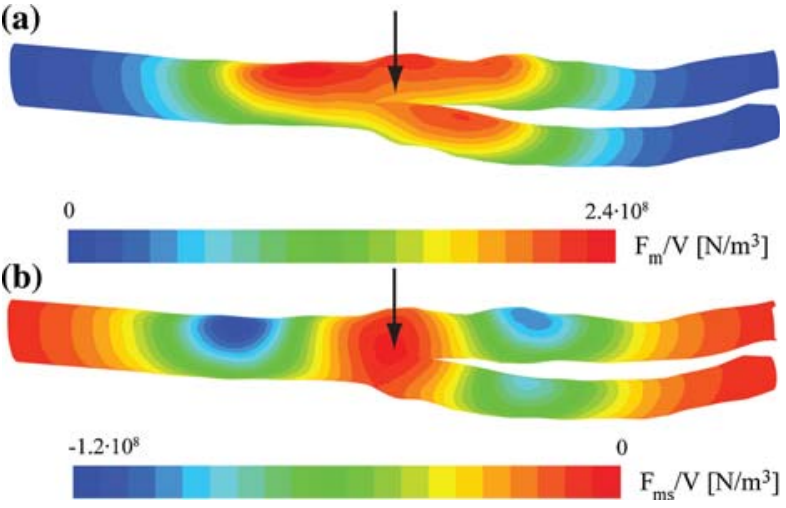

FIGURE 12. The magnetization force per unit volume $\mathrm{F}_{\mathrm{m}} / V$ at the surface of the carotid artery, with (a) the magnitude $F_{\mathrm{m}} / V$ and (b) the cylindrical s-component $F_{\mathrm{ms}} / V$, with $s$ the distance to the axis of symmetry of the cylindrical magnet. The black arrow indicates the target position and orientation of the magnet, located at $d=1 \mathrm{~cm}$ from the target location.

is the reason why the capture efficiency is much higher than for the left coronary artery. The capture efficiency significantly decreases when the magnet distance is increased from 1 to $2 \mathrm{~cm}$ from the target location and is slightly higher when zero velocity after impact with the vessel wall is assumed (inelastic boundary condition). From Fig. 14 the capture efficiency can be seen to vary approximately linearly with the particle diameter $D$, as in the simulations of a cylindrical Poiseuille flow. Apparently, the more complex geometry, the time-dependent flow, and the non-Newtonian viscosity do not change significantly the result $\eta \sim \sqrt{M n_{\mathrm{p}}}$ of Eq. (13).

Figure 15 shows the vertical position at which particles of two different sizes are captured. The small fraction $\eta_{\text {tot }} \approx 15 \%$ of the $250 \mathrm{~nm}$ particles that are captured, have been captured almost homogeneously over the entire domain. The magnet was directed at a vertical position $z \approx 6 \mathrm{~cm} \approx 0.55 z_{\max } \mathrm{cm}$ such that, from Fig. 15, most of the $4 \mu \mathrm{m}$ particles have been captured before the target location. Under the influence of the radial force $F_{\mathrm{ms}}$, shown in Fig. 12, the particles eventually might drift towards the target location. When however the particles are captured in a healthy segment of the artery, not covered with plaque, the particles might, under the influence of the magnetization force, move through the pores in the vessel wall out of the bloodstream. The pores in the endothelium are just small enough to prevent red blood cells to leave the artery, but smaller particles can easily be pulled through by an exerted magnetization force. Using a smaller magnet or changing the orientation of the magnet can result in a better focusing and thereby a more effective treatment. This is an example of how computational simulations can be used as a tool for analysing, in advance, a certain treatment. 

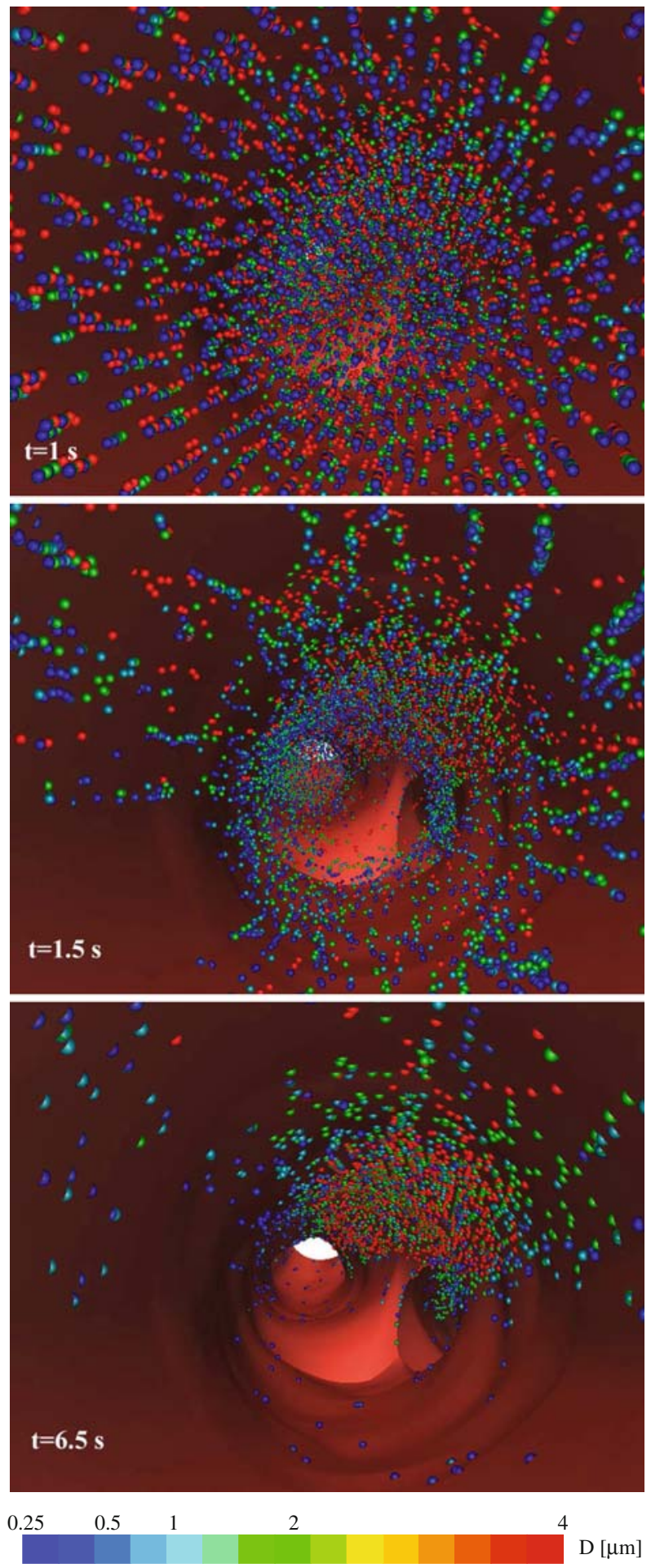

FIGURE 13. Particle positions as seen from inside the main carotid artery at various time instances after the first injection. The particles are colored by their diameter $D$ and not draw to scale. The main carotid artery branches off to the internal carotid artery and to the external carotid artery, the left and right branch respectively. The magnetization force used in these simulations is shown in Fig. 12.

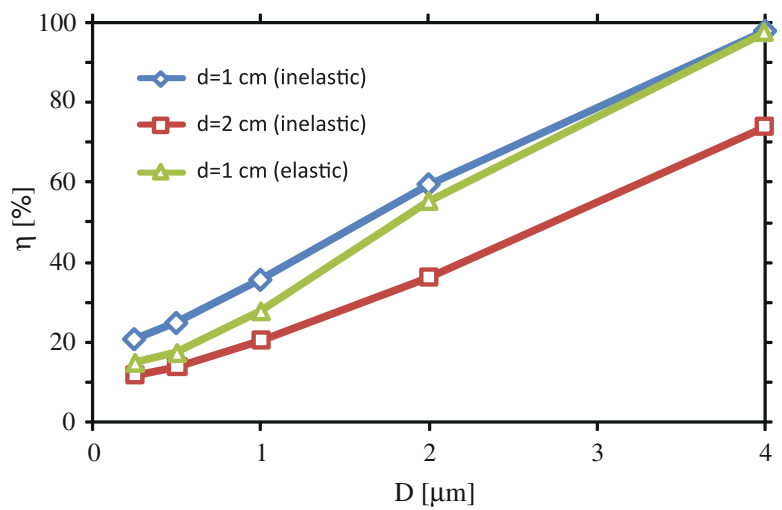

FIGURE 14. The capture efficiency $\eta$ as a function of particle diameter $D$ for two different distances $d$ of the magnet surface to the target location. For $d=1 \mathrm{~cm}$ an inelastic boundary condition for the interaction of particles with the wall is also tested. The magnetization force used in the $d=1 \mathrm{~cm}$ simulations is displayed in Fig. 12.

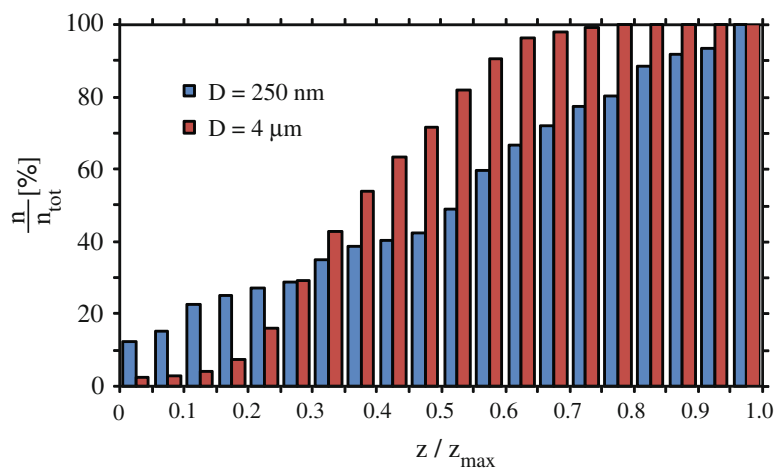

FIGURE 15. A cumulative histogram of the fraction $n / n_{\text {tot }}$ of particles captured in the carotid artery, as a function of the vertical dimensionless capture position $z / z_{\max }$ in the coordinates of Fig. 11a. Here $z_{\max }=11.12 \mathrm{~cm}$ and $n_{\text {tot }}=593$ for the $250 \mathrm{~nm}$ particles and $n_{\mathrm{tot}}=3976$ for the $4 \mu \mathrm{m}$ particles. For these simulations the magnet was placed at a distance of $d=1 \mathrm{~cm}$ and the associated magnetization force is shown in Fig. 12.

\section{DISCUSSION AND CONCLUSIONS}

For negligible particle inertia it has been argued that the particle capture efficiency $\eta$, for $\eta \ll 1$, satisfies the scaling relation $\eta \sim\left(\alpha M n_{\mathrm{p}}\right)^{\beta}$ with $\beta=1 / 2$. Here $\alpha=L / R$ is the ratio between a characteristic value for the spatial extent $L$ of the magnetic field and the vessel radius $R$. The dimensionless 'particle magnetization number' $M n_{\mathrm{p}}=\mu_{0} D^{2} M H / 18 \mu u_{0} L$ is the characteristic ratio between the magnetization force and the drag force. In a simulation of a cylindrical Poiseuille flow, the capture efficiency was found to be approximately proportional to the particle diameter $D$, in agreement with the obtained scaling relation. In a ninety degree 
bended tube, $\beta$ was found to be below or above $1 / 2$ depending on whether the magnetic source was placed more to the inside or to the outside of the bend, respectively.

In simulations of a left coronary artery, only a small fraction of the inserted $2 \mu \mathrm{m}$ iron-carbon magnetic particles could be captured with a superconducting magnet placed externally to the body. This fraction was found to increase approximately fourfold to $25 \%$ upon an increase of the particle diameter from $D=2$ to $4 \mu \mathrm{m}$. This super linear increase was partially due to the fact that the magnetic source was placed on the outside of the coronary artery bend such that $\beta>1 / 2$, but also due to the redirection of particles that would, in the absence of a magnetic field, have left through the circumflex sidebranch.

In the much more superficially located carotid artery more than $50 \%$ of the $2 \mu \mathrm{m}$ particles and almost all of the $4 \mu \mathrm{m}$ particles could be captured with the same magnet positioned at a distance of $1 \mathrm{~cm}$. In this case the capture efficiency was found to be approximately proportional to the particle diameter $D$, in agreement with the obtained scaling relation $\eta \sim \sqrt{\alpha M n_{\mathrm{p}}}$. The fact that the theoretically obtained scaling relation for $\eta \ll 1$ approximately holds up to quite high $\eta$ in this complex geometry with a timedependent velocity and a non-Newtonian viscosity model shows its usefulness and generality. As the simulations of the coronary artery show, however, it must be used with care. Side branches and domain curvature can significantly alter the scaling.

It must be noted that various simplifications have been introduced regarding the geometry, the boundary conditions and the particle modeling. Improvements in the accuracy of the detailed flow pattern are however unlikely to severely alter the results of the simulations. More significant simplifications have been made in the modeling of the drag force. Attached drugs can significantly change the particle shape and effective particle diameter for the drag. Because for a fixed geometry and negligible inertia the only relevant parameter is $M n_{\mathrm{p}}$, one can however simply adjust the present results for such a change in effective diameter. The most severe simplification was made by evaluating the drag force via Stokes' expression, using the macroscopic non-Newtonian fluid viscosity $\mu$. This macroscopic fluid viscosity arises from the complex interaction between the constituents of the blood and is approximately a factor 3.5 higher than the viscosity of the blood plasma alone. Very small particles will move along stream traces in the blood plasma without colliding with blood constituents, therefore experiencing a drag force proportional to the viscosity of the blood plasma. The viscous drag experienced by particles of the order of or smaller than the blood cells, as used in the present work, might therefore be much smaller than that which is presently used by considering the macroscopic viscosity. This would significantly change the outcome of the simulations. The obtained results therefore possibly significantly underestimate the capture efficiency, especially for smaller particles.

Computational simulations, as those discussed in this work, make it possible to study the feasibility of a medical technique before entering clinical trials. Furthermore, simulations are useful for investigating the influence of various factors independently, and for optimization. Later on in the development of a technique simulations are envisioned to aid a doctor by providing on-the-fly numerical experiments. The performed simulations for the coronary and carotid arteries, using present-day materials and magnets, have shown to yield favorable capture efficiencies, justifying further investigation of the considered medical technique. Pre-clinical and clinical studies will have to show whether or not it will turn out to be feasible to use the discussed techniques to treat cardiovascular diseases. It remains, for example, to be seen what effect a strong inhomogeneous magnetic fields has on the functioning of the heart. Since, for effective treatment, the magnetic field has to be applied for quite some time, this is an important issue. This also holds for the study of the removal of the magnetic particles afterwards. From a physics point of view, however, the obtained results are encouraging with regard to the application of the Magnetic Drug Targeting technique to cardiovascular diseases.

\section{ACKNOWLEDGMENTS}

We would like to thank J.V. Soulis of Demokrition University of Thrace for making available to us the mesh of the Left Coronary Artery, and F. Tan and Y. Xu of Imperial College London for the MRI data of the Carotid Artery geometry.

\section{OPEN ACCESS}

This article is distributed under the terms of the Creative Commons Attribution Noncommercial License which permits any noncommercial use, distribution, and reproduction in any medium, provided the original author(s) and source are credited.

\section{REFERENCES}

\footnotetext{
${ }^{1}$ Abramowitz, M., and I. A. Stegun (eds). Handbook of Mathematical Functions with Formulas, Graphs, and Mathematical Tables. New York: Dover, 1972.
} 
${ }^{2}$ Alexiou, C., et al. Locoregional cancer treatment with magnetic drug targeting. Cancer Res. 60:6641-6648, 2000.

${ }^{3}$ Avilés, M. O., et al. Theoretical analysis of a transdermal ferromagnetic implant for retention of magnetic drug carrier particles. J. Magn. Magn. Mat. 293:605-615, 2005.

${ }^{4}$ Aviles, M. O., H. Chen, A. D. Ebner, A. J. Rosengart, M. D. Kaminskic, and J. A. Ritter. In vitro study of ferromagnetic stents for implant assisted-magnetic drug targeting. J. Magn. Magn. Mat. 311:306-311, 2007.

${ }^{5}$ Ballyk, P. D., D. A. Steinman, and C. R. Ethier. Simulation of non-Newtonian blood flow in an end-to-end anastomosis. Biorheology 31(5):565-586, 1994.

${ }^{6}$ Cao, H., G. Huang, S. Xuan, Q. Wu, F. Gu, and C. Li. Synthesis and characterization of carbon-coated iron core/ shell nanostructures. J. Alloys Compd. 448:272-276, 2008. ${ }^{7}$ Chen, H., A. D. Ebner, A. J. Rosengart, A. B. Kaminski, and J. A. Ritter. Analysis of magnetic drug carrier particle capture by a magnetizable intravascular stent: 1. parametric study with single wire correlation. J. Magn. Magn. Mat. 284:181-194, 2004.

${ }^{8}$ Cohen Stuart, D. C. The Development of a Discrete Particle Model for 3D Unstructured Grids: Application to Magnetic Drug Targeting. MSc thesis, Delft University of Technology, 2009.

${ }^{9}$ Decuzzi, P., S. Lee, B. Bhushan, and M. Ferrari. A theoretical model for the margination of particles within blood vessels. Ann. Biomed. Eng. 33(2):179-190, 2005.

${ }^{10}$ Forbe, Z. G., et al. Validation of high gradient magnetic field based drug delivery to magnetizable implants under flow. IEEE Trans. Biomed. Eng. 55:643-649, 2008.

${ }^{11}$ Furlani, E. P., and K. C. Ng. Analytical model of magnetic nanoparticle transport and capture in the microvasculature. Phys. Rev. E 73:061919, 2006.

${ }^{12}$ Giannoglou, G. D., J. V. Soulis, T. M. Farmakis, and G. E. Louridas. Molecular viscosity distribution in the left coronary artery tree. Comp. Cardiol. 30:641-644, 2003.

${ }^{13}$ Grief, A. D., and G. Richardson. Mathematical modelling of magnetically targeted drug delivery. J. Magn. Magn. Mat. 293:455-463, 2005.

${ }^{14}$ Haverkort, J. W., S. Kenjereš, and C. R. Kleijn. Magnetic particle motion in a Poiseuille flow. Phys. Rev. E 80(1): 016302, 2009.

${ }^{15}$ Iacob, Gh., O. Rotariu, N. J. C. Strachan, and U. O. Häfeli. Magnetizable needles and wires: modelling an efficient way to target magnetic microspheres in vivo. Biorheology 41:599-612, 2004.

${ }^{16}$ Johnston, B. M., P. R. Johnston, S. Corney, and D. Kilpatrick. Non-Newtonian blood flow in human right coronary arteries: steady state simulations. J. Biomech. 37:709-720, 2004.

${ }^{17}$ Lübbe, A. S., et al. Preclinical experiences with magnetic drug targeting: tolerance and efficacy. Cancer Res. 56:46944701, 1996.

${ }^{18}$ Lübbe, A. S., et al. Clinical experiences with magnetic drug targeting: a phase i study with 4'-epidoxorubicin in 14 patients with advanced solid tumors. Cancer Res. 56:46864693, 1996.

${ }^{19}$ Lübbe, A. S., et al. Clinical applications of magnetic drug targeting. J. Surg. Res. 95:200-206, 2001.

${ }^{20}$ Marshall, I., P. Papathanasopoulou, and K. Wartolowska. Carotid flow rates and flow division at the bifurcation in healthy volunteers. Physiol. Meas. 25:691-697, 2004.

${ }^{21}$ Morsi, S. A., and A. J. Alexander. An investigation of particle trajectories in two-phase flow systems. J. Fluid Mech. 55(2):193-208, 1972.

${ }^{22}$ Nishijima, S., S.-i. Takeda, F. Mishima, and Y. Tabata. A study of magnetic drug delivery system using bulk high temperature superconducting magnet. IEEE Trans. Appl. Superconduct., 18:870-877, 2008.

${ }^{23}$ Ritter, J. A., A. D. Ebner, K. D. Daniel, and K. L. Stewart. Application of high gradient magnetic separation principles to magnetic drug targeting. J. Magn. Magn. Mat. 280:184 201, 2004.

${ }^{24}$ Sherman, T. F. On connecting large vessels to small. The meaning of Murray's law. J. Gen. Physiol. 30:431-453, 1981.

${ }^{25}$ Smyte, W. R. Static and Dynamic Electricity. New York: McGraw-Hill, 1950.

${ }^{26}$ Soulis, J. V., T. M. Farmakis, G. D. Giannoglou, and G. E. Louridas. Wall shear stress in normal left coronary artery tree. J. Biomech. 39:742-749, 2006.

${ }^{27}$ Takeda, S.-i., F. Mishima, S. Fujimoto, Y. Izumia, and S. Nishijima. Development of magnetically targeted drug delivery system using superconducting magnet. J. Magn. Magn. Mat. 311:367-371, 2007.

${ }^{28}$ Tan, F. P. P., et al. Advanced computational models for disturbed and turbulent flow in stenosed human carotid artery bifurcation. Biomed. Proc. 21:390-394, 2008. 